high incidence of transplacentally induced gliomas and nephroblastomas reported by the experimentalists has attracted much attention from the clinicians. Despite certain defects (notably the lack of an index), the present account provides a timely introduction to what is likely to be a rapidly expanding subject in clinical and experimental oncology.

RICHARD CARTER

Chromosomes in Human Cancer By Jaroslav Cervenka and Lucien Koulischer. Edited by Robert J. Gorlin. (Pp. viii + 203 ; 22 figures. \$13.75.) Springfield, Illinois: Charles C. Thomas. 1973.

The authors have gathered together most of the available information on the chromosome complements of malignant tumours (almost always abnormal) and of leukaemias, benign tumours, and preneoplastic lesions (frequently, though less consistently, abnormal). The first section is devoted to general considerations, including the question of specific changes in solid tumours (the highly consistent loss of a $\mathbf{G}$ group chromosome in meningiomas is not mentioned at this point). There follows a commendable account of the chromosome changes in leukaemias and preleukemic conditions. The final section is devoted to solid tumours.

This is a stimulating and on the whole comprehensible treatise which deals with a large amount of data. Unfortunately, there are many minor errors, particularly misspellings of authors' names, and some that are less trivial. A discussion on clonal evolution is accompanied by a table of karyotypes which may well puzzle the reader (it is not explained that the leukaemic patient concerned had congenital trisomy-21; the source of the table is given as Lejeune et al, 1963, but this paper has been omitted from the references at the end of the chapter). At times, there is confusion over nomenclature. Thus, the terms 'cyst' and 'cystadenoma' are used for malignant tumours, and in a report on ovarian teratomas their benign or malignant status is not given.

N. B. ATKIN

Diagnostic Orthopaedic Pathology By C. G. Woods. (Pp. ix + 298; illustrated. £8.50.) Oxford, London, Edinburgh, Melbourne: Blackwell Scientific Publications. 1972.
This book is intended to be a practical aid to the diagnosis of bone and joint lesions. There is certainly a need for such a book. It is written by the pathologist at the Nuffield Orthopaedic Centre, Oxford, and reflects his extensive experience in the field of pathology as applied to orthopaedics. The author adds his emphasis to the very sound view that diagnosis of many diseases of bones and joints (and presumably of other systems, too) is not to be established on the basis of one piece of evidence alone. Histopathology, radiology, biochemical data, and clinical information are often concerned together, in various combinations, and the wise histologist knows when to call on information from other disciplines at the same time as looking down his microscope.

The question is how the book measures up to its very laudable aim. It is relatively short and easy to read. Its illustrations, from a wide variety of lesions, include many radiographs as well as photomicrographs. One serious defect is the very limited bibliography provided. Another, at least in the experience of the present reviewer, is that the general arrangement of the material is not well suited to the practical diagnostic purpose which is put forward as the chief aim of the book. Perhaps it is likely to be of more use to the undergraduate student who wishes to have a practical account of bone and joint pathology than to the pathologist searching for the solution to a difficult diagnostic problem.

H. A. SISSONS

Muscle Biopsy: A Modern Approach (Major Problems in Neurology, volume 2) By Victor Dubowitz and Michael $\mathbf{H}$. Brooke (Pp. 486; illustrated. £7.50.) London, Philadelphia, Toronto: W. B. Saunders. 1973.

Interpretation of muscle biopsy sections can often pose considerable diagnostic problems for the histopathologist who is not accustomed to dealing with this specialized field. Professor Dubowitz and Dr Brooke are to be congratulated on producing a readable, well illustrated account of the histology of both normal and diseased muscle. The first section of the book is devoted to the techniques used in performing, processing, and staining biopsy material. Histochemical methods are dealt with in some detail and the importance of accurate fibre diameter measurements is stressed. The second section gives a detailed clinico- pathological description of muscle dia orders illustrating the use of these teci: niques, and in particular of enzyme histo? chemistry. The section concludes with am admirable summary of the analytic processes needed to arrive at a diagnos. The third and final section, written by Hans Neville, is devoted to the ultra structural changes in muscle disease and is a first-class, concise account of this rapidly expanding field. This book represents excellent value for money and shoukf find a place on the bookshelf of everg routine histopathology laboratory. M. S. DUNNIII

\section{Medicine and Mycology}

A course on common fungal infection will be held at Fitzwilliam College Cambridge, on 25, 26 and 27 Septemb 1974.

The course is intended for all cliniciar and laboratory workers interested diseases caused by fungi.

The lecturers will include: Professor R. A. Cawson (Guy's Hospital), Dr G. 薃 Clein (St George's Hospital), Dr R. R: Davies (St Mary's Hospital), Dr D. Evans (United Cambridge Hospitals Mr E. G. V. Evans (Leeds Genera Infirmary), Dr Andrew Henderson (Uni, versity Hospital, Cardiff), Dr B. N? Herbertson (Dept. of Pathology, Cambridge), Professor J. R. Hobbs (Wes? minster Hospital), Dr Rosalinde Hurley (Queen Charlotte's Maternity Hospital Dr David Kerridge (Dept. of Bio chemistry, Cambridge), Dr D. W. Ro Mackenzie (London School of Hygieneng Dr M. F. Madelin (Department 90 Botany, Bristol), Dr R. R. Marples (Central Public Health Laboratory, Colip dale), Dr S. O. B. Roberts (United Cambridge Hospitals), Dr Arthur Roow (United Cambridge Hospitals), Mr M. E Snell (St Mary's Hospital), Dr D. C. Ed Speller (United Bristol Hospitals).

Enrolment forms may be obtained from the Secretary, Cambridge Universi Medical School, Hills Road, Cambridge CB2 2QL. 